\title{
Ultrastructural Analysis of Haloalkaliphilic Bacteriophage Isolates from Mono Lake, a North American Soda Lake
}

\author{
K. D. Moulton ${ }^{1}$, A. Hatch ${ }^{1}$, M. Movassaghi ${ }^{1}$, N. Lobo ${ }^{1}$, F. Mwaura ${ }^{2}$, L. J. Rothschild ${ }^{3}$, S. M. Duboise ${ }^{1}$. \\ ${ }^{1}$ University of Southern Maine, Department of Applied Medical Sciences, 96 Falmouth Street, 178 \\ Science Bldg., Portland, ME 04104-9300 \\ ${ }^{2}$ University of Nairobi, Department of Geography and Environmental Studies, University Way, P.O., \\ Box 30197, GPO100, Nairobi, Kenya \\ ${ }^{3}$ NASA Ames Research Center, Mail Stop 239-20, Moffett Field, CA 94035
}

Viruses in soda lakes and hypersaline environments (including some in the western United States such as California's Mono Lake) are known to be highly abundant [1,2,3,4] and have been observed by electron microscopy or pulse-field gel electrophoresis. Cultivation of soda lake bacteriophages followed by genomic sequencing and annotation in preparation for further genetic functional analysis has been rare [5]. Despite microbial biodiversity projects, including the Mono Lake Microbial Observatory (http://www.monolake.uga.edu/), cultured viral isolates are few and viral genomics data associated with virus host systems from North American soda lakes have been largely lacking. Here we present an initial ultrastructural survey of bacteriophages that were isolated and cultivated from Mono Lake during the summer and fall seasons of 2012. The numerous bacteriophage isolates obtained and the diverse morphotypes shown in Figure 1 are consistent with the expected high bacteriophage abundance and diversity. Genomic sequencing and initial structural protein studies are in progress. As discussed further below, these data are expected to permit comparative genomic analysis of bacteriophages infecting similar microbes in geographically separated soda lakes in North America and East Africa.

In prior and continuing studies we isolated bacteriophages and their microbial hosts from soda lakes of the Great Rift Valley in Kenya which are among the most alkaline environments on Earth [5,6]. We have fully sequenced and annotated the genomes of several of the soda lake phages from Kenya and have noted interesting conserved genomic sequences of unknown function which are present in otherwise distinct phages from at least two of the East African lakes [5]. The phage genomes have numerous open reading frames including those in the observed conserved region that are currently of unknown function. It is of interest to determine whether Mono Lake phage genomes will reveal genetic elements in common with those of the Kenyan phage genomes that may be specifically relevant to the biology of the bacteriophages in these distinct but similarly hypersaline and alkaline environments. Patterns of genomic conservation of similar genes are being used in targeting specific open reading frames for determinations of whether the genes may serve functions essential for virus replication or relevant to adaptation to hypersaline and alkaline environments.

The images in Figure 1 show bacteriophages of diverse morphotypes isolated from Mono Lake. Preliminary host strain identifications have been obtained by 16S rRNA gene PCR amplification and sequence analysis. Comparative studies of virus structural composition, capsid self-assembly, and morphogenesis are of particular interest in the haloalkaliphilic environment that requires the phages to assemble under intracellular $\mathrm{pH}$ and ionic conditions and then remain stable and infectious in the much more saline and alkaline extracellular soda lake environments. Evolutionary comparisons and investigations of viral structural proteins and the biochemical determinants of their capsid self-assembly and stability within cells and within ecosystems are major topics of particular interest. As reported 
previously, we are exploring the capsid-based self assembling nanoparticle structures of selected soda lake phages and their potential for novel applications in nanomedicine [6]. The support of funding agencies and institutional support of the University of Southern Maine (USM) are gratefully acknowledged [7].

\section{References:}

[1] S. Jiang, et al. 2003. Microb. Ecol. 47: 9-17

[2] S. Sabet, et al. 2006. Microb. Ecol. 51:543-554.

[3] J. R. Brum, and G. F. Steward. 2010. Microb. Ecol. 60: 636-643.

[4] Y. Bettarel, et al. 2011. FEMS Micrbiol. Ecol. 76: 360-372.

[5] N. Lobo-Galo 2013. Ph.D. Dissertation, University of Maine.

[6] N. Lobo et al. 2012. Microscopy and Microanalysis, 18, (Suppl 2), 110-111.

[7] The authors acknowledge funding by a Maine Space Grant Consortium 2012 Seed Grant, NASA EPSCoR RID EP-11-01, EP-12-01, a NSF GK-12 international supplement DGE-0749059, USM graduate fellowships for A. Hatch, M. Movassaghi, and N Lobo, and a NSF GK-12 Fellowship (DGE0440560) for M. Movassaghi. USM and ongoing funded projects sustain TEM resources established originally by NSF MRI (CNS-0521262) funding.

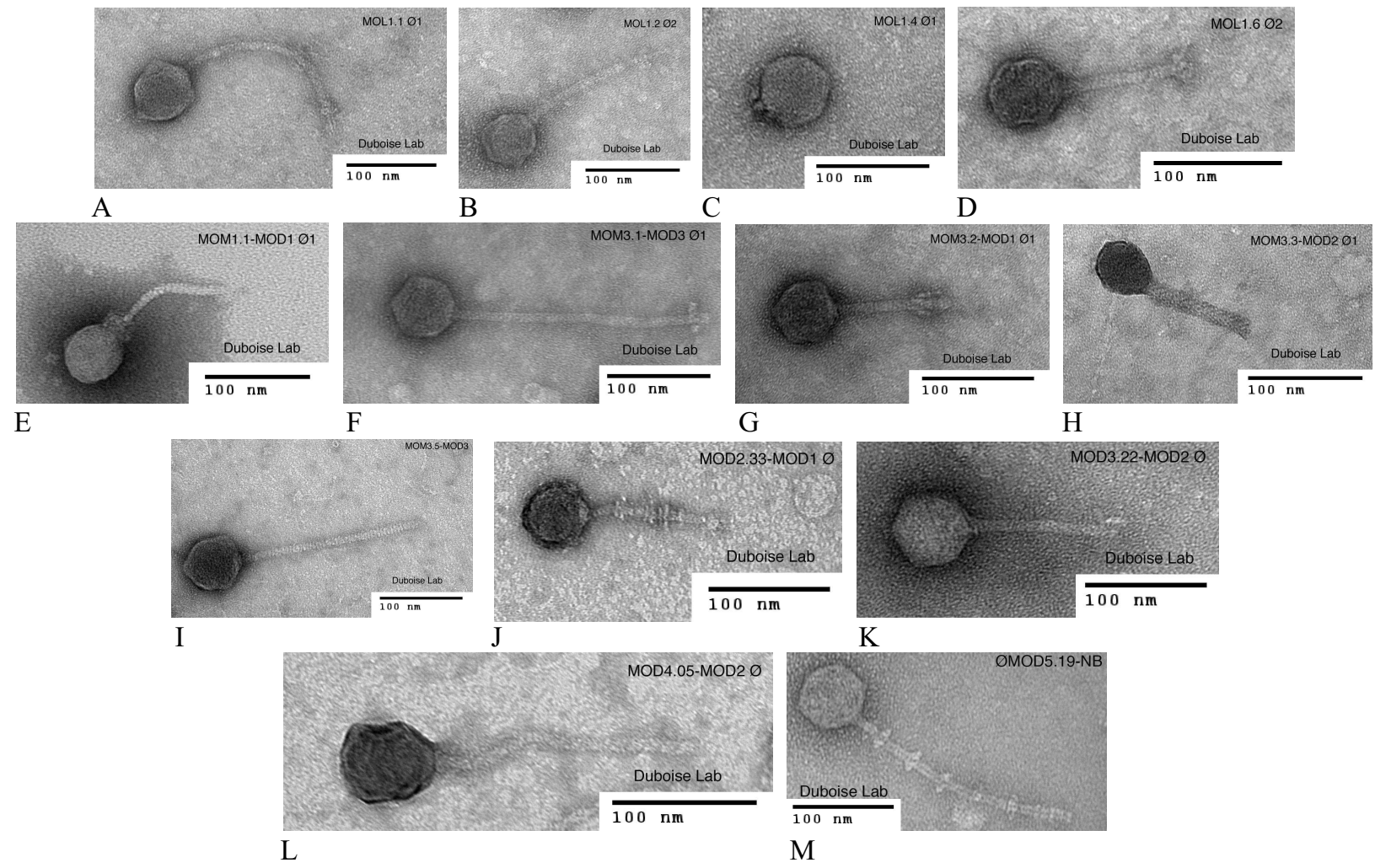

Figure 1. Shown are initial images of selected bacteriophages isolated from Mono Lake water samples during 2012. Morphotypes are consistent with classification in the Order Caudovirales, the tailed phages, and include representatives of the virus families Podoviridae (e.g., panel C), Siphoviridae (e.g., panels A, B, D, E, F, G, I, J, K, L, M), and Myoviridae (e.g., H). Bacteriophages were negatively stained using 2\% UA and subsequently imaged using USM's transmission electron microscope, a FEI TECNAI G2 Spirit BioTWIN operating at 100kV. 